\title{
Logarithm laws for strong unstable foliations in negative curvature and non-Archimedian Diophantine approximation
}

\author{
Jayadev S. Athreya and Frédéric Paulin
}

\begin{abstract}
Given a finite volume negatively curved Riemannian manifold $M$, we give a precise relation between the logarithmic growth rates of the excursions of the strong unstable leaves of negatively recurrent unit tangent vectors into cusp neighborhoods of $M$ and their linear divergence rates under the geodesic flow. Our results hold in the more general setting where $M$ is the quotient of any proper CAT $(-1)$ metric space $X$ by any geometrically finite discrete group of isometries of $X$. As an application to non-Archimedian Diophantine approximation in positive characteristic, we relate the growth of the orbits of $\mathscr{O}_{\widehat{K}}$-lattices under one-parameter unipotent subgroups of $\mathrm{GL}_{2}(\hat{K})$ with approximation exponents and continued fraction expansions of elements of the local field $\widehat{K}$ of formal Laurent series over a finite field.
\end{abstract}

Mathematics Subject Classification (2010). 37D40, 53D25, 11J61, 11J70, 20E08, $20 \mathrm{G} 25$.

Keywords. Negative curvature, geodesic flow, horocyclic flow, strong unstable foliation, cusp excursions, logarithm law, Diophantine approximation, continued fraction, approximation exponent.

\section{Introduction}

In the seminal paper [Sul], D. Sullivan proved what he coined a logarithm law governing the statistical behavior of excursions of geodesic flow lines to cusps in a noncompact, finite volume Riemannian manifold $M$ with constant negative sectional curvature: if $o: T^{1} M \rightarrow M$ is the unit tangent bundle, then for every $x \in M$, and almost every (with respect to Lebesgue's measure) $v \in T^{1} M$, we have

$$
\limsup _{t \rightarrow \infty} \frac{d\left(o\left(\phi_{t} v\right), x\right)}{\log t}=\frac{1}{n-1}
$$

where $\left(\phi_{t}\right)_{t \in \mathbb{R}}$ is the geodesic flow on $T^{1} M$ and $d$ is the hyperbolic distance on $M$.

This result has inspired a significant body of work over the last 30 years on the excursions of geodesic flow lines into neighborhoods of infinity in negatively curved manifolds. Probabilistic aspects have been considered by, for example, Enriquez, Franchi, Guivarc'h, Le Jan [EFJ]. In more geometric directions, Sullivan's result 
has been extended by Kleinbock-Margulis [KIM] to finite volume locally symmetric spaces of non-compact type, by Stratmann-Velani [SV] to geometrically finite constant negative curvature, by Hersonsky-Paulin [HP4], [HP5] to variable negative curvature and to trees, and by Athreya-Ghosh-Prasad [AGP1], [AGP2] to some buildings.

Closely related to geodesic flows are horocycle flows and their generalizations. In 2-dimension hyperbolic geometry, the horocycle flow is a flow on the unit tangent bundle of a hyperbolic surface, whose orbits are the leaves of the strong unstable foliation of the geodesic flow. More generally, the ergodic theory and topological dynamics of the strong unstable foliation (also called the horospherical foliation) have been the subject of significant foundational works in dynamical systems. In the setting of negative curvature, seminal contributions have been made by, among others, Hedlund, Furstenberg, Dal'Bo, Dani, Roblin, Sarig, Schapira, Smillie, as well as the recent Chapter 9 of [PPS]. In higher rank, the field has been transformed in particular by the work of Ratner, with fundamental contributions by, among many others, Dani, Dani-Margulis, Kleinbock-Margulis, Margulis, Margulis-Tomanov, and more recently Benoist-Quint. See for example the survey [Esk] for a more complete bibliography.

Much of the above study has focused on rigidity phenomena (for example classification of orbit closures and invariant measures). In the non-compact setting, this often involved studying non-divergence phenomena, in particularly understanding that measures supported on leaves of the strong unstable foliation satisfy appropriate tightness conditions. In contrast, Sullivan-type questions about the excursions of horocyclic flow lines or of the leaves of the strong unstable foliation to neighborhoods of infinity in non-compact spaces have only recently been explored, first in the work of Athreya-Margulis [AM] for unipotent or horospherical actions in some locally symmetric spaces of non-compact type, and also in [Ath], [KeM].

In this paper, we are interested in this problem of excursions of (projections of) horospheres into cuspidal neighborhoods, and applications to Diophantine approximation, a component which is present in many of the previous works. We work in the very general setting of quotients $M=\Gamma \backslash X$ of proper CAT(-1) metric spaces $X$ by geometrically finite groups of isometries $\Gamma$ (we will recall all necessary material about the now well-known notion of geometrical finiteness). In Subsection 1.1, we give a simplified version of our main theorem, when $M$ is a (geometrically finite) negatively curved Riemannian manifold. We stress the fact that no constant curvature (nor locally symmetric) assumption is made.

As allowed by our general setting, an example of particular interest is when $X$ is the Bruhat-Tits tree for the group $\mathrm{GL}_{2}(\widehat{K})$, where $\widehat{K}$ is the field of formal Laurent series over a finite field. Here, we will obtain our main Diophantine applications, precise relationships between the asymptotics of excursions near infinity withapproximation exponents and continued fraction expansions of elements of the field $\widehat{K}$, which we describe below in Subsection 1.2. 
1.1. Riemannian manifolds. Let $M$ be a complete, geometrically finite (for instance finite volume), Riemannian manifold with dimension at least 2 and sectional curvature at most -1 . Let $o: T^{1} M \rightarrow M$ be its unit tangent bundle and $\left(\phi_{t}\right)_{t \in \mathbb{R}}$ its geodesic flow. For every $v \in T^{1} M$, let

$$
W^{\mathrm{su}}(v)=\left\{w \in T^{1} M: \lim _{t \rightarrow+\infty} d\left(o\left(\phi_{-t} v\right), o\left(\phi_{-t} w\right)\right)=0\right\}
$$

be the strong unstable leaf of $v$, endowed with the naturally scaling Hamenstädt's distance $d^{\text {su }}$ (see Section 2, it coincides with the induced Riemannian distance when the sectional curvature is constant) and, when non-compact, with the filter of the complementary subsets of its relatively compact subsets. We say that $v \in T^{1} M$ is negatively recurrent under the geodesic flow if there are a compact set $K$ in $T^{1} M$ and a sequence of times $\left(t_{n}\right)_{n \in \mathbb{N}}$ converging to $-\infty$ such that $\phi_{t_{n}} v \in K$. When $M$ has finite volume, this excludes only countably many orbits of (compact) strong unstable leaves under the geodesic flow.

Our main result, Theorem 7, whose simplified version is given below, is a precise relation between the logarithmic growth rates of the strong unstable foliation and the linear divergence rates of the geodesic flow.

Theorem 1. For every $v \in T^{1} M$ which is negatively recurrent under the geodesic flow, we have

$$
\limsup _{w \in W^{\mathrm{su}}(v)} \frac{d(o(w), o(v))}{\log d^{\mathrm{su}}(w, v)}=1+\limsup _{t \rightarrow+\infty} \frac{\left.d\left(o\left(\phi_{-t} v\right), o(v)\right)\right)}{t} .
$$

In the particular case when $M$ is a finite volume orientable hyperbolic surface, we recover, by a purely geometric proof, the logarithm law for the excursions into cusp neighborhoods of the horocyclic flow due to Theorem 2.10 of [Ath], generalizing the work of $[\mathrm{AM}]$ on $\mathrm{SL}_{2}(\mathbb{Z}) \backslash \mathbb{H}_{\mathbb{R}}^{2}$. In particular, in this setting we have, by an application of the easy half of the Borel-Cantelli lemma and an estimate on volumes, that for almost every $v$,

$$
\limsup _{t \rightarrow+\infty} \frac{\left.d\left(o\left(\phi_{-t} v\right), o(v)\right)\right)}{t}=0,
$$

and so

$$
\limsup _{w \in W^{\mathrm{su}}(v)} \frac{d(o(w), o(v))}{\log d^{\mathrm{su}}(w, v)}=1
$$

for almost every $v$. We remark that in [AM] there is a factor of $1 / 2$ which is present due to the fact that in that paper $T^{1}\left(\mathrm{SL}_{2}(\mathbb{Z}) \backslash \mathbb{H}_{\mathbb{R}}^{2}\right)=\mathrm{SL}_{2}(\mathbb{Z}) \backslash \mathrm{SL}_{2}(\mathbb{R})$ was viewed as the space of unimodular lattices in $\mathbb{R}^{2}$, and that the depth of cusp excursions was measured by the (logarithm) of the length of the shortest vector in the lattice. See [Ath] for further discussion.

Claim 5.3 in [Ath] strengthens the result of Theorem 2.10 of [Ath] by allowing one to specify which cuspidal neighborhoods one is interested in visiting. Our results 
also allow us to specify a set of cusps into whose neighborhoods we want to study the excursions of the strong unstable manifolds, as follows. Recall that a cusp of $M$ is an asymptotic class of minimizing geodesic rays in $M$ along which the injectivity radius tends to 0 . For every cusp $e$, let $r_{e}:[0,+\infty[\rightarrow M$ be a representative of $e$, and let $\beta_{e}: M \rightarrow\left[0,+\infty\left[\right.\right.$ be the map $x \mapsto \max \left\{0, \lim _{t \rightarrow+\infty} t-d\left(x, r_{e}(t)\right)\right\}$. (One way to normalize $\beta_{e}$ is to ask for $r_{e}$ being contained in the closure of, and start from the boundary of, a maximal open Margulis neighborhood of $e$ (see for instance [BK], [Bow], [HP4], it is a canonical neighborhood of the end of $M$ to which converges $e$ if $M$ has finite volume).) Given a (necessarily finite since $M$ is geometrically finite) set $E$ of cusps, let $\beta_{E}=\max _{e \in E} \beta_{e}$.

Theorem 2. For every $v \in T^{1} M$ which is negatively recurrent under the geodesic flow, we have

$$
\limsup _{w \in W^{\mathrm{su}}(v)} \frac{\beta_{E}(o(w))}{\log d^{\mathrm{su}}(w, v)}=1+\limsup _{t \rightarrow+\infty} \frac{\beta_{E}\left(o\left(\phi_{-t} v\right)\right)}{t} .
$$

Note that the above statements are stronger than almost-everywhere statements, as for instance when $M$ has constant curvature and finite volume, the right-hand side may take any value in $[1,+\infty$ [, but is 1 for almost every $v$, by Sullivan's result. We refer to Corollary 12 for almost everywhere consequences of these theorems for the excursions of the strong unstable leaves in cusp neighborhoods.

As already mentioned, these theorems are valid when $M$ is replaced by the quotient of any proper CAT $(-1)$ metric space $X$ by any geometrically finite discrete group of isometries of $X$, see Section 4.

1.2. Diophantine approximation. We now give an application of our main result to non-Archimedian Diophantine approximation in positive characteristic (see for instance [Las], [Sch] for nice introductions).

Let $k=\mathbb{F}_{q}$ be a finite field with $q$ elements, where $q$ is a positive power of a prime $p$. Let $A=k[X]$ be the ring of polynomials in one variable $X$ over $k$ and let $K=k(X)$ be its fraction field, endowed with the absolute value $|\cdot|=|\cdot|_{\infty}$ defined by

$$
\left|\frac{P}{Q}\right|=q^{\operatorname{deg} P-\operatorname{deg} Q} .
$$

Let $\widehat{K}$ be the completion of $K$ for this absolute value, which is the field $k\left(\left(X^{-1}\right)\right)$ of formal Laurent series $f=\sum_{i \in \mathbb{Z}} f_{i} X^{-i}$ (where $f_{i} \in k$ is zero for $i \in \mathbb{Z}$ small enough), with absolute value $|\cdot|=|\cdot|_{\infty}$ defined by

$$
|f|=q^{-\sup \left\{j \in \mathbb{Z}: f_{i}=0 \text { for all } i<j\right\}} .
$$

For every $f \in \hat{K}-K$, the approximation exponent $v=v(f)$ of $f$ is the least upper 
bound of the positive numbers $v^{\prime}$ such that there exist infinitely many elements $\frac{P}{Q}$ in $K$ (with $P, Q$ relatively prime) such that

$$
\left|f-\frac{P}{Q}\right| \leq|Q|^{-v^{\prime}}
$$

Artin's continued fraction expansion of $f \in \widehat{K}-K$ is the sequence $\left(a_{i}=a_{i}(f)\right)_{i \in \mathbb{N}}$ in $A$ with $\operatorname{deg} a_{i}>0$ if $i>0$ such that

$$
f=a_{0}+\frac{1}{a_{1}+\frac{1}{a_{2}+\frac{1}{a_{3}+\frac{1}{\ddots}}}} .
$$

Let $\mathscr{O}=k\left[\left[X^{-1}\right]\right]$ be the local ring of formal power series $f=\sum_{i \in \mathbb{N}} f_{i} X^{-i}$ (where $\left.f_{i} \in k\right)$ in $X^{-1}$ over $k$. An $\mathscr{O}$-lattice is a free $\mathscr{O}$-submodule of rank 2 in the $\widehat{K}$-vector space $\widehat{K}^{2}$. The linear action of $\mathrm{GL}_{2}(\widehat{K})$ on $\widehat{K}^{2}$ induces an action of $\mathrm{GL}_{2}(\widehat{K})$ on the set of $\mathscr{O}$-lattices. For every $\mathscr{O}$-lattice $\Lambda$, let $\Delta(\Lambda)$ be the unique $n \in \mathbb{N}$ such that there exists $\gamma \in \mathrm{SL}_{2}(A)$ and $\lambda \in \widehat{K}$ such that $\lambda \gamma \Lambda=\mathscr{O} \times X^{-n} \mathscr{O}$. For every $f \in \widehat{K}-K$, let $\left(u_{g}=u_{g}(f)\right)_{g \in \hat{K}}$ be the maximal one-parameter unipotent subgroup of $\operatorname{SL}_{2}(\hat{K})$ whose projective action on the projective line $\mathbb{P}_{1}(\widehat{K})=\widehat{K} \cup\{\infty\}$ fixes $f$.

Using the approach in [Pau1] and the Bruhat-Tits building of $\left(\mathrm{PGL}_{2}, \widehat{K}\right)$, we have the following result, relating, for a given irrational formal Laurent series $f$, the logarithmic growth of the orbit of any $\mathscr{O}$-lattice under the one-parameter unipotent subgroup of $\mathrm{SL}_{2}(\hat{K})$ fixing $f$ with the approximation exponent of $f$ and with the continued fraction expansion of $f$.

Theorem 3. For every $f \in \widehat{K}-K$, we have

$$
\limsup _{|g| \rightarrow+\infty} \frac{\Delta\left(u_{g} \mathscr{O}^{2}\right)}{\log _{q}|g|}=2-\frac{2}{v}=1+\limsup _{n \rightarrow+\infty} \frac{\log \left|a_{n}\right|}{\log \left|a_{n} \prod_{i=0}^{n-1} a_{i}^{2}\right|} .
$$

We give versions of this result for Diophantine approximation with congruence conditions in Section 5.

Acknowledgement. We thank the Mathematisches Forschungsinstitut Oberwolfach, where this project was started. The first author thanks the Université Paris-Sud (Orsay) where this paper was continued, and G. A. Margulis for many useful and inspiring discussions. The first author also would like to acknowledge the support of NSF grant DMS 1069153. The second author thanks the Mittag-Leffler Institute where this paper was completed, and J. Parkkonen for the discussion of Corollary 13. 


\section{Background on CAT(-1) spaces}

We refer to $[\mathrm{BH}]$ for the definitions and basic properties of CAT(-1) spaces, and the knowledgeable reader may skip this section.

Let $(X, d)$ be a proper CAT $(-1)$ geodesic metric space, and $\bar{X}^{\text {geo }}=X \cup \partial_{\infty} X$ its cone-topology compactification by the asymptotic classes of its geodesic rays.

We denote by $T^{1} X$ the space of geodesic lines in $X$, that is, of isometric maps $v: t \mapsto v_{t}$ from $\mathbb{R}$ into $X$. To simplify the notation, we will denote by $v_{t}$ instead of $v(t)$ the image by $v \in T^{1} X$ of $t \in \mathbb{R}$. When $X$ is a (complete, simply connected) Riemannian manifold (with dimension at least 2 and sectional curvature at most -1 ), this notation coincides with the usual one, upon identifying a unit tangent vector and the geodesic line it defines. We denote by $v_{ \pm} \in \partial_{\infty} X$ the points at $\pm \infty$ of any $v \in T^{1} X$. The geodesic flow $\left(\phi_{t}\right)_{t \in \mathbb{R}}$ is the action of $\mathbb{R}$ on $T^{1} X$ by translations at the source: $\phi_{t} v: s \mapsto v_{s+t}$ for all $s, t \in \mathbb{R}$ and $v \in T^{1} X$.

The Busemann cocycle is the continuous map $\beta: \partial_{\infty} X \times X \times X \rightarrow \mathbb{R}$, defined by

$$
(\xi, x, y) \mapsto \beta_{\xi}(x, y)=\lim _{t \rightarrow+\infty} d\left(x, \xi_{t}\right)-d\left(y, \xi_{t}\right),
$$

where $t \mapsto \xi_{t}$ is any geodesic ray converging to $\xi$. For every $\xi \in \partial_{\infty} X$, the horospheres centered at $\xi$ are the level sets $f^{-1}(\lambda)$ for $\lambda \in \mathbb{R}$ of the map $f: y \mapsto$ $\beta_{\xi}\left(y, x_{0}\right)$ from $X$ to $\mathbb{R}$, and the (closed) horoballs centered at $\xi$ are its sublevel sets $\left.\left.f^{-1}(]-\infty, \lambda\right]\right)$ for $\lambda \in \mathbb{R}$, for some (hence any) $x_{0} \in X$.

If $C$ is a nonempty closed convex subset of $X$ and $\xi \in \partial_{\infty} X-\partial_{\infty} C$, the closest point to $\xi$ on $C$ is the unique point of $C$ which minimizes the map $y \mapsto \beta_{\xi}\left(y, x_{0}\right)$, for some (hence any) given $x_{0} \in X$.

Let $\Gamma$ be a discrete group of isometries of $X$. We denote by $\pi: X \rightarrow \Gamma \backslash X$ the canonical projection of $X$ onto its quotient metric space $\Gamma \backslash X$, whose distance is again denoted by $d$.

The limit set of $\Gamma$ will be denoted by $\Lambda \Gamma$, and the convex hull of this limit set by $\mathscr{C} \Lambda \Gamma$. Recall that $\Gamma$ is nonelementary if $\operatorname{Card}(\Lambda \Gamma) \geq 3$. The conical limit set $\Lambda_{c} \Gamma$ of $\Gamma$ is the set of points $\xi \in \partial_{\infty} X$ such that there exists a sequence of orbit points of some (hence any) $x_{0} \in X$ under $\Gamma$ converging to $\xi$ while staying at bounded distance from a geodesic ray converging to $\xi$. The points in $\Lambda_{c} \Gamma$ are called the conical limit points.

A point $p \in \partial_{\infty} X$ is a bounded parabolic point of $\Gamma$ if it is the fixed point of a parabolic element of $\Gamma$ and if its stabilizer $\Gamma_{p}$ in $\Gamma$ acts properly with compact quotient on $\Lambda \Gamma-\{p\}$. A discrete nonelementary group of isometries $\Gamma$ of $X$ is called geometrically finite if every element of $\Lambda \Gamma$ is either a conical limit point or a bounded parabolic point of $\Gamma$.

When $X$ is the real hyperbolic $n$-space $\mathbb{H}_{\mathbb{R}}^{n}$ and $\Gamma$ is torsion-free, this is equivalent to the fact that $\Gamma$ has a fundamental domain bounded by finitely many totally geodesic hyperplanes. But the works of Thurston and many others have shown that this definition is not ideal to work with, and we refer to [Bow] for foundational results 
concerning the various definitions. In particular, when $X$ is Riemannian (without any assumption of constant curvature), $\Gamma$ is geometrically finite if and only if the quotient by $\Gamma$ of the 1-neighborhood of the convex hull in $X$ of the limit set of $\Gamma$ has finite volume.

Let $\operatorname{Par}_{\Gamma}$ be the set of fixed points of parabolic elements of $\Gamma$. If $\Gamma$ is a geometrically finite group of isometries of $X$, then (see for instance [Bow]) the action of $\Gamma$ on $\operatorname{Par}_{\Gamma}$ has only finitely many orbits, and there exists a $\Gamma$-equivariant family $\left(\mathrm{HB}_{p}\right)_{p \in \mathrm{Par}_{\Gamma}}$ of pairwise disjoint closed horoballs, with $\mathrm{HB}_{p}$ centered at $p$, such that the quotient

$$
\Gamma \backslash\left(\mathscr{C} \Lambda \Gamma-\bigcup_{p \in \operatorname{Par}_{\Gamma}} \mathrm{HB}_{p}\right)
$$

is compact, and any geodesic ray from the boundary of $\mathrm{HB}_{p}$ to $p$ injects isometrically by the canonical projection $\pi: X \rightarrow \Gamma \backslash X$.

For every $v \in T^{1} X$, the strong unstable leaf of $v$ is

$$
W^{\mathrm{su}}(v)=\left\{w \in T^{1} X: \lim _{t \rightarrow+\infty} d\left(v_{-t}, w_{-t}\right)=0\right\} .
$$

The set $\left\{w_{0}: w \in W^{\mathrm{su}}(v)\right\}$ is exactly the horosphere centered at $v_{-}$through $v_{0}$.

For every $v \in T^{1} X$, let $d^{\text {su }}$ be Hamenstädt's distance on the strong unstable leaf of $v$, defined as follows (see [HP1], Appendix, compare with [Ham], see also $\$ 2.2$ of [HP6] for a generalisation when horoballs are replaced by arbitrary nonempty closed convex subsets): for all $w, w^{\prime} \in W^{\mathrm{su}}(v)$,

$$
d^{\mathrm{su}}\left(w, w^{\prime}\right)=\lim _{t \rightarrow+\infty} e^{\frac{1}{2} d\left(w-t, w_{-t}^{\prime}\right)-t} .
$$

This limit exists, and Hamenstädt's distance is a distance inducing the original topology on $W^{\text {su }}(v)$. We will denote by $B^{\text {su }}(w, r)$ the ball of center $w$ and radius $r$ in the metric space $\left(W^{\mathrm{su}}(v), d^{\mathrm{su}}\right)$. For all $t \in \mathbb{R}$ and $w, w^{\prime} \in W^{\mathrm{su}}(v)$, and for every isometry $\gamma$ of $X$, we have $\gamma W^{\mathrm{su}}(v)=W^{\mathrm{su}}(\gamma v), \phi_{t} W^{\mathrm{su}}(v)=W^{\mathrm{su}}\left(\phi_{t} v\right)$, $d^{\mathrm{su}}\left(\gamma w, \gamma w^{\prime}\right)=d^{\mathrm{su}}\left(w, w^{\prime}\right)$ and

$$
d^{\mathrm{su}}\left(\phi_{t} w, \phi_{t} w^{\prime}\right)=e^{t} d^{\mathrm{su}}\left(w, w^{\prime}\right) .
$$

Remarks. (1) When $X$ is a Riemannian manifold with constant sectional curvature, then Hamenstädt's distance is the induced Riemannian distance on the horosphere of base points of vectors of $W^{\mathrm{su}}(v)$ (see for instance [HP2]). When $X$ is a complex hyperbolic space $\mathbb{H}_{\mathbb{C}}^{n}$, then Hamenstädt's distance is a multiple of Cygan's distance, see $\$ 3.11$ of [HP3].

(2) When $X$ is a metric tree, then $d^{\text {su }}(v, w)=\min \left\{t \in \mathbb{R}: v_{-t}=w_{-t}\right\}$.

(3) Here is a coarse interpretation of Hamenstädt's distance. Let $\kappa>0$ be fixed. Let $\tau$ be the map defined on the set of couples of elements of $T^{1} X$ in the same strong unstable leaf, with values in $[0,+\infty[$, by

$$
\tau(v, w)=\min \left\{t \in \mathbb{R}: d\left(v_{-t}, w_{-t}\right) \leq \kappa\right\} .
$$


Then is its easy to prove that there exists a constant $c \geq 0$, depending only on $\kappa$, such that

$$
\left|\log d^{\mathrm{su}}(v, w)-\tau(v, w)\right| \leq c .
$$

Finally, we denote by $\log$ the natural $\log a r i t h m$, with $\log (e)=1$.

\section{Excursions in horospheres}

In this section we give the geometric lemmas concerning the behavior of horospheres that we will need to prove our main theorem. We refer for instance to [PP1] for more information on the excursion properties of geodesic lines in convex subsets of CAT (-1) spaces.

Let $X$ be a CAT(-1) geodesic metric space. We will use several times without mention the first of the following lemmas, which is well known and follows by comparison with a geodesic triangle with an obtuse angle in the real hyperbolic plane $\mathbb{H}_{\mathbb{R}}^{2}$. In all the following pictures, a white dot represents a point at infinity of $X$.

Lemma 4. Let $x \in X$ and $y, z \in X \cup \partial_{\infty} X$ be such that $x$ is the closest point to $z$ on $[x, y]$. Let $q^{\prime} \in[x, z]$ and let $q$ be the intersection point of $[y, z]$ with the sphere or horosphere centered at $z$ and passing through $q^{\prime}$. Then

$$
\begin{gathered}
d(x,[y, z]) \leq c_{1}=\log (1+\sqrt{2}), \\
d\left(q, q^{\prime}\right) \leq c_{2}=2 \log \frac{1+\sqrt{3}}{2} .
\end{gathered}
$$

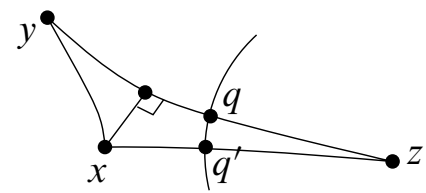

For instance by Lemma 2.9 of [PP1], for every horosphere $H$ with center $\xi$, for every $\eta \in \partial_{\infty} X-\{\xi\}$, for every $x, y \in H$ such that the geodesic rays $[x, \eta[$ and $[y, \eta[$ meet $H$ only at $x$ and $y$ respectively, we have

$$
d(x, y) \leq 2 c_{1} .
$$

Lemma 5. Let $x, y \in X$ be two points in a horosphere centered at $\xi \in \partial_{\infty} X$, let $z$ be the closest point to $\xi$ on $[x, y]$, and let $z^{\prime}$ be the closest point to $y$ on $[x, \xi[$. Then

$$
|d(x, z)-d(y, z)| \leq 2 c_{1} \text { and }\left|d\left(z^{\prime}, x\right)-d\left(z^{\prime}, y\right)\right| \leq c_{2} .
$$


Proof. This is well known, we only prove the second statement. If $\xi_{t}$ is the point at distance $t$ from $x$ on $\left[x, \xi\right.$ [, and if $q_{t}$ is the intersection with $\left[y, \xi_{t}\right]$ of the sphere centered at $\xi_{t}$ through $z^{\prime}$, then $d\left(z^{\prime}, q_{t}\right) \leq c_{2}$ by Lemma 4 and

$$
\left|d\left(z^{\prime}, x\right)-d\left(z^{\prime}, y\right)\right| \leq\left|d\left(z^{\prime}, x\right)-d\left(q_{t}, y\right)\right|+d\left(z^{\prime}, q_{t}\right),
$$

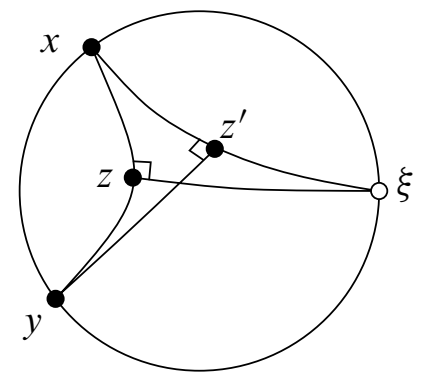

and $\lim _{t \rightarrow+\infty} d\left(z^{\prime}, x\right)-d\left(q_{t}, y\right)=\beta_{\xi}(x, y)=0$.

Lemma 6. Let $v \in T^{1} X$ and $w \in W^{\mathrm{su}}(v)$. If $d^{\mathrm{su}}(v, w)>e^{\frac{c_{2}}{2}}$, then the closest point $v_{t_{w}}$ to $w_{+}$on the geodesic line ] $v_{-}, v_{+}\left[\right.$belongs to the geodesic ray ] $\left.v_{-}, v_{0}\right]$, and

$$
\left|\log d^{\mathrm{su}}(v, w)-\right| t_{w}|| \leq \frac{8 c_{1}+c_{2}}{2} .
$$

Proof. By the triangle inequality, we have

$$
d^{\mathrm{su}}(v, w) \leq e^{\frac{1}{2} d\left(v_{0}, w_{0}\right)} .
$$

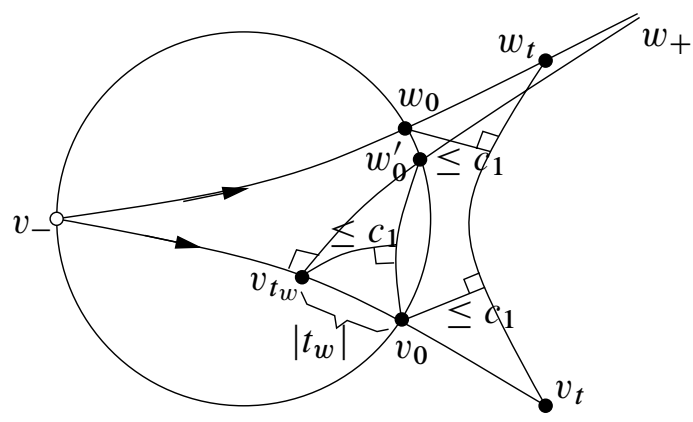

If $v_{t_{w}}$ does not belong to the geodesic ray ] $\left.v_{-}, v_{0}\right]$, then $d\left(v_{0}, w_{0}\right) \leq c_{2}$ by Lemma 4 , which contradicts the assumption that $d^{\text {su }}(v, w)>e^{\frac{c_{2}}{2}}$. Let $w_{0}^{\prime}$ be the intersection point of the geodesic ray $\left[v_{t_{w}}, w_{+}\left[\right.\right.$and the horosphere $o\left(W^{\mathrm{su}}(v)\right)$. Then, 
using Equation (2) for the second equality, and Lemma 5 for the last equality, writing $A=B \pm C$ instead of $|A-B| \leq C$, we have, for $t \geq 0$,

$$
\begin{aligned}
d\left(v_{t}, w_{t}\right)-2 t & =d\left(v_{0}, w_{0}\right) \pm 4 c_{1}=d\left(v_{0}, w_{0}^{\prime}\right) \pm 6 c_{1} \\
& =d\left(v_{0}, v_{t_{w}}\right)+d\left(v_{t_{w}}, w_{0}^{\prime}\right) \pm 8 c_{1} \\
& =2\left|t_{w}\right| \pm\left(8 c_{1}+c_{2}\right) .
\end{aligned}
$$

By dividing by 2 and by taking the limit as $t$ tends to $+\infty$, this proves the result.

\section{Horospherical logarithm laws}

Let $X$ be a proper CAT $(-1)$ geodesic metric space, let $\Gamma$ be a geometrically finite group of isometries of $X$, and let $\pi: X \rightarrow \Gamma \backslash X$ be the canonical projection. For all $v \in T^{1} X$, consider the nondecreasing map $\Theta_{v}:[0,+\infty[\rightarrow[0,+\infty[$ defined by

$$
\Theta_{v}(s)=\sup _{w \in B^{\mathrm{su}}(v, s)} d\left(\pi\left(w_{0}\right), \pi\left(v_{0}\right)\right) .
$$

A map $\psi:] 0,+\infty[\rightarrow] 0,+\infty$ [ will be called slowly increasing if $t \mapsto \psi(t)$ and $t \mapsto \frac{t}{\psi(t)}$ are nondecreasing for $t$ big enough, if $\lim _{t \rightarrow+\infty} \psi(t)=+\infty$, and if $\lim _{t \rightarrow+\infty} \frac{\psi(t+c)}{\psi(t)}=1$ for all $c \in \mathbb{R}$. Let $a_{\psi}=\lim _{t \rightarrow+\infty} \frac{t}{\psi(t)} \in[0,+\infty]$. For instance, for all $a>0$ and $\alpha \in] 0,1]$, the map $t \mapsto a t^{\alpha}$ is slowly increasing with $a_{\psi}=a$ if $\alpha=1$ and $a_{\psi}=+\infty$ otherwise. From now on, we fix such a map $\psi$. We use the convention that $+\infty+t=+\infty$ for all $t \in[0,+\infty]$. Note that $\psi(t) \sim \frac{1}{a_{\psi}} t$ as $t \rightarrow+\infty$ if $a_{\psi} \neq 0,+\infty$, where $f(t) \sim g(t)$ as $t \rightarrow+\infty$ is Landau's usual notation for $f(t)-g(t)=\mathrm{o}(g(t))$ as $t \rightarrow \infty$.

Theorem 7. For all $v \in T^{1} X$ such that $v_{-}$is a conical limit point of $\Gamma$, we have

$$
\limsup _{s \rightarrow+\infty} \frac{\Theta_{v}(s)}{\psi(\log s)}=a_{\psi}+\limsup _{t \rightarrow+\infty} \frac{d\left(\pi\left(v_{-t}\right), \pi\left(v_{0}\right)\right)}{\psi(t)} .
$$

Proof. We start the proof by making some reductions. Let us fix $v \in T^{1} X$ and denote by $\mathscr{H}_{v}$ the horosphere with center $v_{-}$through $v_{0}$. Let $\left(\mathrm{HB}_{p}\right)_{p \in \operatorname{Par}_{\Gamma}}$ be a $\Gamma$-equivariant family of pairwise disjoint closed horoballs as in Section 2, let $H_{p}=\partial \mathrm{HB}_{p}$ be the horosphere bounding $\mathrm{HB}_{p}$, and let $X_{\mathrm{Par}}=\bigcup_{p \in \operatorname{Par}_{\Gamma}} \stackrel{\circ}{\mathrm{HB}_{p}}$ be the union of the interiors of the horoballs $\mathrm{HB}_{p}$ for $p \in \operatorname{Par}_{\Gamma}$. Let $\Delta$ be the diameter of $\Gamma \backslash\left(\mathscr{C} \Lambda \Gamma-X_{\mathrm{Par}}\right)$.

Since $\Lambda \Gamma$ has no isolated point and since $v_{-} \in \Lambda \Gamma$, the strong unstable leaf $W^{\text {su }}(v)$ in $T^{1} X$ is non-compact. Indeed, if $\left(\xi_{n}\right)_{n \in \mathbb{N}}$ is a sequence of points in $\Lambda \Gamma-\left\{v_{-}\right\}$ converging to $v_{-}$, then the geodesic line from $v_{-}$to $\xi_{n}$ meets the horosphere $o\left(W^{\text {su }}(v)\right)$ in exactly one point $x_{n}$, and the elements $v_{n} \in T^{1} X$ such that $v_{n}(0)=x_{n},\left(v_{n}\right)_{-}=$ 
$v_{-}$and $\left(v_{n}\right)_{+}=\xi_{n}$ are points in $W^{\text {su }}(v)$ which as $n \rightarrow+\infty$ go out of every compact subset of $W^{\mathrm{su}}(v)$. We endow $W^{\text {su }}(v)$ with the filter of the complementary subsets of its relatively compact subsets, and we will consider limits and upper limits of functions defined on $W^{\text {su }}(v)$ along this filter. What we have to prove is

$$
\limsup _{w \in W^{\mathrm{su}}(v)} \frac{d\left(w_{0}, \Gamma v_{0}\right)}{\psi\left(\log d^{\mathrm{su}}(w, v)\right)}=a_{\psi}+\limsup _{t \rightarrow+\infty} \frac{d\left(v_{-t}, \Gamma v_{0}\right)}{\psi(t)} .
$$

Since $\psi$ is slowly increasing and by the triangle inequality, the validity of this formula is unchanged if we replace $v$ by any other given element of $W^{\text {su }}(v)$. We may hence assume that $v_{0} \in \mathscr{C} \Lambda \Gamma$ (note that $\Lambda \Gamma$ is not reduced to $\left\{v_{-}\right\}$). Since $v_{-} \in \Lambda \Gamma$, the negative geodesic ray $v_{]-\infty, 0]}$ is therefore contained in $\mathscr{C} \Lambda \Gamma$. Since $\psi$ is slowly increasing, by Equation (1) and by the triangle inequality, the validity of Equation (3) is unchanged by replacing $v$ by $\phi_{-t_{0}} v$ for any fixed $t_{0} \geq 0$. Since $v_{-}$is a conical limit point, we may thus assume that $v_{0} \in \mathscr{C} \Lambda \Gamma-X_{\mathrm{Par}}$.

Let us now introduce some more notation.
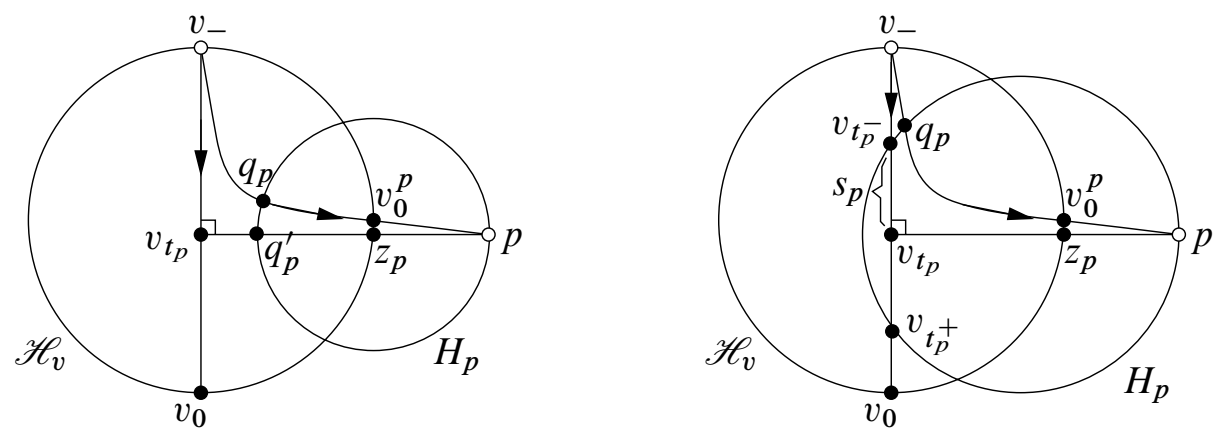

For every $p \in \operatorname{Par}_{\Gamma}$, let $\left.\left.t_{p} \in\right]-\infty, 0\right]$ be such that $v_{t_{p}}$ is the closest point to $p$ on the geodesic ray $v_{]-\infty, 0]}$, let $z_{p}$ be the intersection point with $\mathscr{H}_{v}$ of the geodesic ray $\left[v_{t_{p}}, p\right.$ [ , let $q_{p}$ be the closest point to $v_{-}$on $H_{p}$, and let $v^{p}$ be the unique element of $W^{\text {su }}(v)$ such that $v_{+}^{p}=p$. Note that the intersection with $\mathscr{H}_{v}$ of the geodesic line $v^{p}$ is its time 0 point $v_{0}^{p}$. For all $p \in \operatorname{Par}_{\Gamma}$ such that $v_{t_{p}} \notin \mathrm{HB}_{p}$, let $q_{p}^{\prime}$ be the closest point to $v_{t_{p}}$ on $H_{p}$. For all $p \in \operatorname{Par}_{\Gamma}$ such that $v_{t_{p}} \in \mathrm{HB}_{p}$, let $\left.\left.t_{p}^{ \pm} \in\right]-\infty, 0\right]$ be such that $v_{t_{p}^{-}}$(respectively $v_{t_{p}^{+}}$) is the entering (respectively exiting) point of the geodesic line $v$ in (respectively out) of $\mathrm{HB}_{p}$, and let $s_{p}=t_{p}-t_{p}^{-} \geq 0$.

By Lemma 6, for every $p \in \operatorname{Par}_{\Gamma}$ such that $d^{\text {su }}\left(v, v^{p}\right)>e^{\frac{c_{2}}{2}}$, we have

$$
\left|\log d^{\mathrm{su}}\left(v, v^{p}\right)-\right| t_{p}|| \leq \frac{8 c_{1}+c_{2}}{2} .
$$

By the initial reduction, we have $d\left(v_{t_{p}^{-}}, \Gamma x_{0}\right) \leq \Delta, t_{p}^{+} \leq 0$ and $d\left(v_{t_{p}^{+}}, \Gamma x_{0}\right) \leq \Delta$ for every $p \in \operatorname{Par}_{\Gamma}$ such that $v_{t_{p}} \in \mathrm{HB}_{p}$. The following estimate will also be useful. 
Lemma 8. Let $p \in \operatorname{Par}_{\Gamma}$. If $v_{t_{p}} \in \mathrm{HB}_{p}$, then

$$
\left|d\left(v_{0}^{p}, \Gamma v_{0}\right)-\left(\left|t_{p}\right|+s_{p}\right)\right| \leq 5 c_{1}+c_{2}+\Delta .
$$

If $v_{t_{p}} \notin \mathrm{HB}_{p}$, then

$$
\left|d\left(v_{0}^{p}, \Gamma v_{0}\right)-\left(\left|t_{p}\right|-d\left(v_{t_{p}}, \mathrm{HB}_{p}\right)\right)\right| \leq 2 c_{1}+2 c_{2}+\Delta .
$$

Proof. If $v_{t_{p}} \in \mathrm{HB}_{p}$, since the geodesic ray $\left[q_{p}, p\right.$ [ isometrically injects in $\Gamma \backslash X$ and since $q_{p}$ belongs to $\mathscr{C} \Lambda \Gamma$ and is the closest point to $v_{0}^{p}$ on $H_{p}$, by Equation (2), and by the second part of Lemma 5 for the last equality, writing $A=B \pm C$ instead of $|A-B| \leq C$, we have

$$
\begin{aligned}
d\left(v_{0}^{p}, \Gamma v_{0}\right) & =d\left(v_{0}^{p}, q_{p}\right) \pm \Delta \\
& =d\left(z_{p}, v_{t_{p}}\right) \pm\left(\Delta+4 c_{1}\right) \\
& =d\left(z_{p}, v_{t_{p}}\right)+d\left(v_{t_{p}}, v_{t_{p}^{-}}\right) \pm\left(\Delta+5 c_{1}\right) \\
& =\left|t_{p}\right|+s_{p} \pm\left(\Delta+5 c_{1}+c_{2}\right) .
\end{aligned}
$$

The proof of the second assertion is similar: If $v_{t_{p}} \notin \mathrm{HB}_{p}$, then

$$
\begin{aligned}
d\left(v_{0}^{p}, \Gamma v_{0}\right) & =d\left(v_{0}^{p}, q_{p}\right) \pm \Delta \\
& =d\left(z_{p}, q_{p}^{\prime}\right) \pm\left(\Delta+2 c_{1}+c_{2}\right) \\
& =d\left(z_{p}, v_{t_{p}}\right)-d\left(v_{t_{p}}, q_{p}^{\prime}\right) \pm\left(\Delta+2 c_{1}+c_{2}\right) \\
& =\left|t_{p}\right|-d\left(v_{t_{p}}, \mathrm{HB}_{p}\right) \pm\left(\Delta+2 c_{1}+2 c_{2}\right) .
\end{aligned}
$$

Now that the notation is in place, let us prove Equation (3) by reducing both sides to computations inside the horoballs $\mathrm{HB}_{p}$, using the above notation.

Let us endow the set $\operatorname{Par}_{\Gamma}$, and any infinite subset of it, with the Fréchet filter of the complementary subsets of its finite subsets. We also consider limits and upper limits of functions defined on this set along this filter. We denote by $\mathrm{HB}_{p}$ the interior of $\mathrm{HB}_{p}$.

Lemma 9. If $\left\{p \in \operatorname{Par}_{\Gamma}: v_{t_{p}} \in \stackrel{\circ}{\mathrm{HB}}_{p}\right\}$ is finite, then $\lim \sup _{t \rightarrow+\infty} \frac{d\left(v_{-t}, \Gamma v_{0}\right)}{\psi(t)}=0$, and otherwise

$$
\limsup _{t \rightarrow+\infty} \frac{d\left(v_{-t}, \Gamma v_{0}\right)}{\psi(t)}=\limsup _{p \in \operatorname{Par}_{\Gamma}: v_{t_{p}} \in \mathrm{HB}_{p}} \frac{s_{p}}{\psi\left(\left|t_{p}\right|\right)} .
$$

Proof. For all $t \in]-\infty, 0]$ such that $v_{t} \notin X_{\mathrm{Par}}=\bigcup_{p \in \operatorname{Par}_{\Gamma}} \stackrel{\circ}{\mathrm{HB}_{p}}$, we have $d\left(v_{t}, \Gamma v_{0}\right) \leq \Delta$, which in particular proves the first claim, since $\lim _{t \rightarrow+\infty} \psi(t)=$ $+\infty$. Let $p \in \operatorname{Par}_{\Gamma}$ be such that $v_{t_{p}} \in \mathrm{HB}_{p}$. For all $t \in\left[t_{p}^{-}, t_{p}\right]$, let $r$ be the closest point to $v_{t}$ on the geodesic ray $\left[v_{t_{p}^{-}}, p[\right.$, see the picture below. 


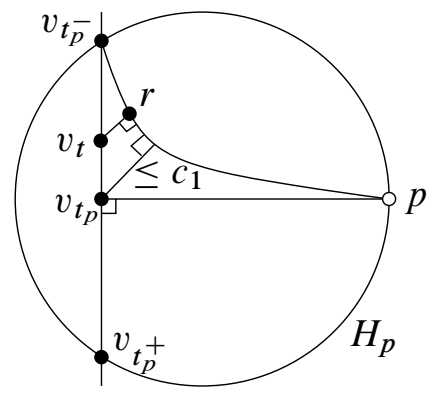

Since $d\left(v_{t_{p}},\left[v_{t_{p}^{-}}, p[) \leq c_{1}\right.\right.$ and by convexity, since the geodesic ray $\left[v_{t_{p}}, p[\right.$ isometrically injects in $\Gamma \backslash X$ and since $v_{t_{p}}$ belongs to $\mathscr{C} \Lambda \Gamma$ and is the closest point to $r$ on $H_{p}$, writing $A=B \pm C$ instead of $|A-B| \leq C$, we have

$$
\begin{aligned}
d\left(v_{t}, \Gamma v_{0}\right) & =d\left(r, \Gamma v_{0}\right) \pm c_{1} \\
& =d\left(r, v_{t_{p}^{-}}\right) \pm\left(c_{1}+\Delta\right) \\
& =d\left(v_{t}, v_{t_{p}^{-}}\right) \pm\left(2 c_{1}+\Delta\right) \\
& =\left|t-t_{p}^{-}\right| \pm\left(2 c_{1}+\Delta\right) .
\end{aligned}
$$

Similarly, for all $t \in\left[t_{p}, t_{p}^{+}\right]$, we have $\left|d\left(v_{t}, \Gamma v_{0}\right)-\right| t-t_{p}^{+}|| \leq 2 c_{1}+\Delta$.

Since $t \mapsto \psi(t)$ and $t \mapsto \frac{t}{\psi(t)}$ are eventually nondecreasing, if $\left|t_{p}\right|$ is big enough, note that $\frac{\left|t-t_{p}^{-}\right|}{\psi(|t|)}=\frac{t-t_{p}^{-}}{\psi(-t)}$ is maximal as $t$ ranges in $\left.] t_{p}^{-}, t_{p}\right]$ when $t=t_{p}$, and $\frac{\left|t-t_{p}^{+}\right|}{\psi(|t|)}=$ $\frac{t_{p}^{+}-t}{\psi(-t)}$ is maximal as $t$ ranges in $\left[t_{p}, t_{p}^{+}\left[\right.\right.$also when $t=t_{p}$. Since $\left|d\left(v_{t_{p}}, \Gamma v_{0}\right)-s_{p}\right| \leq$ $2 c_{1}+\Delta$, and ||$t_{p}-t_{p}^{-}\left|-s_{p}\right| \leq 2 c_{1}$ by Lemma 5 , this proves the result.

Lemma 10. If $\left\{p \in \operatorname{Par}_{\Gamma}: v_{t_{p}} \in \stackrel{\circ}{\mathrm{HB}}_{p}\right\}$ is finite, then

$$
\limsup _{w \in W^{\mathrm{su}}(v)} \frac{d\left(w_{0}, \Gamma v_{0}\right)}{\psi\left(\log d^{\mathrm{su}}(w, v)\right)}=a_{\psi},
$$

and otherwise

$$
\limsup _{w \in W^{\mathrm{su}}(v)} \frac{d\left(w_{0}, \Gamma v_{0}\right)}{\psi\left(\log d^{\mathrm{su}}(w, v)\right)}=a_{\psi}+\limsup _{p \in \operatorname{Par}_{\Gamma}: v_{t_{p}} \in \mathrm{HB}_{p}} \frac{s_{p}}{\psi\left(\left|t_{p}\right|\right)} .
$$

Proof. Since $v_{-} \in \Lambda_{c} \Gamma$, there exist $C>0$ and a sequence $\left(\gamma_{n}\right)_{n \in \mathbb{N}}$ in $\Gamma$ such that $\lim _{n \rightarrow+\infty} d\left(v_{0}, \gamma_{n} v_{0}\right)=+\infty$ and $\sup _{n \in \mathbb{N}} d\left(\gamma_{n} v_{0}, v_{]-\infty, 0]}\right) \leq C$. In particular, since the family of pairwise disjoint horoballs $\left(\mathrm{HB}_{p}\right)_{p \in \operatorname{Par}_{\Gamma}}$ is locally finite in $X$, there exists a sequence $\left(p_{n}\right)_{n \in \mathbb{N}}$ in $\operatorname{Par}_{\Gamma}$ such that $\lim _{n \rightarrow+\infty} t_{p_{n}}=-\infty$ and $\sup _{n \in \mathbb{N}} d\left(v_{t_{p_{n}}}, \mathrm{HB}_{p_{n}}\right) \leq C+\Delta$. By Equation (4) and Lemma 8, since $\psi$ is eventually nondecreasing, for all $p \in \operatorname{Par}_{\Gamma} \operatorname{such}$ that $d^{\mathrm{su}}\left(v, v^{p}\right)$ is big enough, we 
have

$$
\begin{aligned}
& \frac{d\left(v_{0}^{p}, \Gamma v_{0}\right)}{\psi\left(\log d^{\mathrm{su}}\left(v^{p}, v\right)\right)} \\
& \quad \geq \min \left\{\frac{\left|t_{p}\right|-d\left(v_{t_{p}}, \mathrm{HB}_{p}\right)-2 c_{1}-2 c_{2}-\Delta}{\psi\left(\left|t_{p}\right|+\frac{8 c_{1}+c_{2}}{2}\right)}, \frac{\left|t_{p}\right|-5 c_{1}-c_{2}-\Delta}{\psi\left(\left|t_{p}\right|+\frac{8 c_{1}+c_{2}}{2}\right)}\right\} .
\end{aligned}
$$

In particular,

$$
\limsup _{w \in W^{\text {su }}(v)} \frac{d\left(w_{0}, \Gamma v_{0}\right)}{\psi\left(\log d^{\mathrm{su}}(w, v)\right)} \geq \limsup _{n \rightarrow+\infty} \frac{d\left(v_{0}^{p_{n}}, \Gamma v_{0}\right)}{\psi\left(\log d^{\operatorname{su}}\left(v^{p_{n}}, v\right)\right)} \geq a_{\psi} .
$$

Let $w \in W^{\mathrm{su}}(v)$ with $d^{\mathrm{su}}(w, v)>e^{\frac{c_{2}}{2}}$. Let $v_{t_{w}}$ be the closest point to $w_{+}$on the geodesic line $v$, which belongs to $v_{-\infty, 0}$ by Lemma 6 (see the picture below). Let $z_{w}$ be the intersection point with $\mathscr{H}_{v}$ of the geodesic ray $\left[v_{t_{w}}, p[\right.$, which satisfies $d\left(z_{w}, w_{0}\right) \leq 2 c_{1}$ by Equation (2).

If $v_{t_{w}} \notin X_{\mathrm{Par}}=\bigcup_{p \in \operatorname{Par}_{\Gamma}} \stackrel{\circ}{\mathrm{HB}}_{p}$, then respectively by the triangle inequality, since $v_{t_{w}} \in \mathscr{C} \Lambda \Gamma$, by the second claim of Lemma 5, and by Lemma 6, we have

$$
\begin{aligned}
d\left(w_{0}, \Gamma v_{0}\right) & \leq d\left(z_{w}, \Gamma v_{0}\right)+2 c_{1} \\
& \leq d\left(z_{w}, v_{t_{w}}\right)+2 c_{1}+\Delta \leq\left|t_{w}\right|+2 c_{1}+c_{2}+\Delta \\
& \leq \log d^{\mathrm{su}}(w, v)+\frac{12 c_{1}+3 c_{2}}{2}+\Delta .
\end{aligned}
$$

In particular, if $\left\{p \in \operatorname{Par}_{\Gamma}: v_{t_{p}} \in \stackrel{\circ}{\mathrm{HB}}_{p}\right\}$ is finite, since $\psi$ is slowly increasing, we have

$$
\limsup _{w \in W^{\text {su }}(v)} \frac{d\left(w_{0}, \Gamma v_{0}\right)}{\psi\left(\log d^{\mathrm{su}}(w, v)\right)} \leq \limsup _{w \in W^{\text {su }}(v)} \frac{\log d^{\mathrm{su}}(w, v)}{\psi\left(\log d^{\mathrm{su}}(w, v)\right)}
$$

is at most $a_{\psi}$, hence is equal to $a_{\psi}$ by Equation (5). This proves the first claim of Lemma 10.

We may hence assume that $\left\{p \in \operatorname{Par}_{\Gamma}: v_{t_{p}} \in \stackrel{\circ}{\mathrm{HB}_{p}}\right\}$ is infinite. In particular,

$$
\begin{aligned}
\limsup _{w \in W^{\mathrm{su}}(v)} \frac{d\left(w_{0}, \Gamma v_{0}\right)}{\psi\left(\log d^{\mathrm{su}}(w, v)\right)} & =\limsup _{w \in W^{\mathrm{su}}(v): v_{t_{w}} \in X_{\mathrm{Par}}} \frac{d\left(w_{0}, \Gamma v_{0}\right)}{\psi\left(\log d^{\mathrm{su}}(w, v)\right)} \\
& \geq \limsup _{p \in \operatorname{Par}_{\Gamma}: v_{t_{p}} \in \mathrm{HB}_{p}} \frac{d\left(v_{0}^{p}, \Gamma v_{0}\right)}{\psi\left(\log d^{\mathrm{su}}\left(v^{p}, v\right)\right)} .
\end{aligned}
$$

Let us prove that the converse inequality holds. Since $\psi$ is slowly increasing, by Equation (4) and the first part of Lemma 8, this will prove Lemma 10.

Let $w \in W^{\mathrm{su}}(v)$ and $p \in \operatorname{Par}_{\Gamma}$ such that $d^{\mathrm{su}}(w, v)>e^{\frac{c_{2}}{2}}$ and $v_{t_{w}} \in \stackrel{\circ}{\mathrm{HB}_{p}}$. In particular, $v_{t_{p}} \in \mathrm{HB}_{p}$. Assume for instance that $v_{t_{w}} \in\left[v_{t_{p}}, v_{t_{p}^{+}}[\right.$. 
Let $z_{w}^{\prime}$ be the intersection point with $\mathscr{H}_{v}$ of the geodesic ray $\left[v_{t_{p}^{+}}, p[\right.$, which satisfies $d\left(z_{w}^{\prime}, w_{0}\right) \leq 2 c_{1}$ by Equation (2). Then, again using the second claim of Lemma 5 for the final inequality,

$$
\begin{aligned}
d\left(w_{0}, \Gamma v_{0}\right) & \leq d\left(z_{w}^{\prime}, \Gamma v_{0}\right)+2 c_{1} \leq d\left(z_{w}^{\prime}, v_{t_{p}^{+}}\right)+2 c_{1}+\Delta \\
& \leq d\left(z_{w}, v_{t_{p}^{+}}\right)+4 c_{1}+\Delta \\
& \leq d\left(z_{w}, v_{t_{w}}\right)+d\left(v_{t_{w}}, v_{t_{p}^{+}}\right)+4 c_{1}+\Delta \\
& \leq\left|t_{w}\right|+\left|t_{w}-t_{p}^{+}\right|+4 c_{1}+c_{2}+\Delta .
\end{aligned}
$$

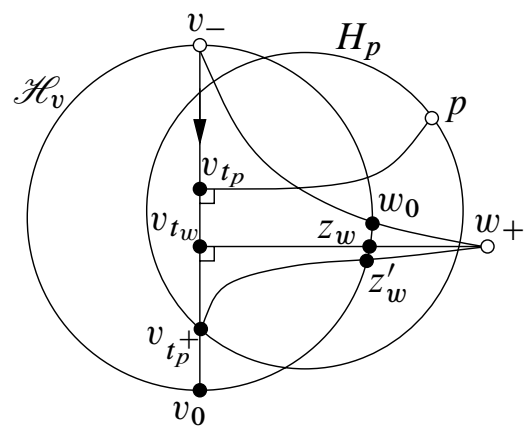

The map from $\left[t_{p}, t_{p}^{+}\left[\right.\right.$to $\left[0,+\infty\left[\right.\right.$ defined by $t_{w} \mapsto \frac{\left|t_{w}\right|+\left|t_{w}-t_{p}^{+}\right|}{\psi\left(\left|t_{w}\right|\right)}=\frac{t_{p}^{+}-2 t_{w}}{\psi\left(-t_{w}\right)}$ is nonincreasing if $t_{p}^{+}$is small enough, with maximum reached at $t_{w}=t_{p}$. This maximum is at least $\frac{\left|t_{p}\right|+s_{p}-2 c_{1}}{\psi\left(\left|t_{p}\right|\right)}$ by the first assertion of Lemma 5. Since $\lim _{t \rightarrow+\infty} \psi(t)=+\infty$, this proves the converse inequality of Equation (6) by the first part of Lemma 8, thus proves Lemma 10.

Now Equation (3), hence Theorem 7, follows immediately from Lemma 9 and Lemma 10.

For every $p \in \operatorname{Par}_{\Gamma}$, let $\tilde{\beta}_{p}: X \rightarrow[0,+\infty[$ be the (well-defined and 1-Lipschitz) map

$$
\tilde{\beta}_{p}: x \mapsto \max \left\{0, \lim _{t \rightarrow+\infty} t-d\left(x, r_{p}(t)\right)\right\},
$$

where $r_{p}:\left[0,+\infty\left[\rightarrow X\right.\right.$ is any geodesic ray from a point of $H_{p}$ to $p$. For every $\Gamma$-invariant subset $E$ of $\operatorname{Par}_{\Gamma}$, let

$$
\tilde{\beta}_{E}=\max _{p \in E} \tilde{\beta}_{p},
$$

which is a $\Gamma$-invariant 1-Lipschitz map from $X$ to $[0,+\infty[$. The proof of the following result is the same as the one of Theorem 7, up to replacing the full family $\left(\mathrm{HB}_{p}\right)_{p \in \operatorname{Par}_{\Gamma}}$ by the subfamily $\left(\mathrm{HB}_{p}\right)_{p \in E}$. 
Theorem 11. For every $\Gamma$-invariant subset $E$ of $\operatorname{Par}_{\Gamma}$, for every $v \in T^{1} X$ such that $v_{-} \in \Lambda_{c} \Gamma$, we have

$$
\limsup _{w \in W^{\mathrm{su}}(v)} \frac{\tilde{\beta}_{E}\left(w_{0}\right)}{\psi\left(\log d^{\mathrm{su}}(w, v)\right)}=a_{\psi}+\limsup _{t \rightarrow+\infty} \frac{\tilde{\beta}_{E}\left(v_{-t}\right)}{\psi(t)} .
$$

Theorem 2 in the introduction is a corollary of Theorem 11, since replacing $\tilde{\beta}_{p}$ by $\tilde{\beta}_{p}+c_{p}$ for any constant $c_{p} \in \mathbb{R}$ depending only on the orbit of $p$ under $\Gamma$ does not change its validity.

Note that under the hypothesis of an almost sure logarithm law for an invariant measure of the geodesic flow, the right-hand sides of the equations in Theorem 7 and Theorem 11 are an easily computed constant for almost every $v$, hence so are the left-hand sides. In particular, the next result follows from Corollary 6.1 of [HP4] and Corollary 1.2 of [HP5]. The new assumptions are satisfied in particular if the Riemannian metric of $X$ is locally symmetric in at least one horoball centered at each parabolic point. We refer for instance to [Rob] for the definitions and properties of the critical exponents and of the Bowen-Margulis measure.

Corollary 12. Let $(X, \Gamma)$ be as above, with $\operatorname{Par}_{\Gamma}$ nonempty. Let $\delta$ be the critical exponent of $\Gamma$. Assume furthermore that either $X$ is a locally finite tree, or $X$ is a Riemannian manifold with pinched sectional curvature such that, for every $p \in \operatorname{Par}_{\Gamma}$, if $\delta_{p}$ is the critical exponent of the stabilizer $\Gamma_{p}$ of $p$ in $\Gamma$, then $\delta_{p}<\delta$ and there exists $c>0$ such that $\frac{1}{c} e^{\delta_{p} n} \leq \operatorname{Card}\left\{\alpha \in \Gamma_{p}: d\left(x_{0}, \alpha x_{0}\right) \leq n\right\} \leq c e^{\delta_{p} n}$ for all $n \in \mathbb{N}$.

Then for almost every $v \in T^{1} X$ for the Bowen-Margulis measure of $\Gamma$ on $T^{1} X$, we have

$$
\limsup _{s \rightarrow+\infty} \frac{\Theta_{v}(s)}{\psi(\log s)}=a_{\psi}
$$

Proof. Let $\delta_{0}=\max _{p \in \operatorname{Par}_{\Gamma}} \delta_{p}<\delta$.

First note that, by $\S 2$ of [Rob] the set of elements $v \in T^{1} X$ such that $v_{+} \in \Lambda_{c} \Gamma$ has full measure for the Bowen-Margulis measure. Also note that since $\psi$ is slowly increasing, if $a_{\psi} \neq+\infty$, then $\lim _{t \rightarrow+\infty} \frac{\log t}{\psi(t)}=0$. Hence if $a_{\psi} \neq+\infty$, by Theorem 7, we have, for almost every $v \in T^{1} X$ for the Bowen-Margulis measure, if $\lim \sup _{t \rightarrow+\infty} \frac{d\left(v_{-t}, \Gamma v_{0}\right)}{\log t}$ is finite, then

$$
\limsup _{s \rightarrow+\infty} \frac{\Theta_{v}(s)}{\psi(\log s)}=a_{\psi}+\limsup _{t \rightarrow+\infty} \frac{d\left(v_{-t}, \Gamma v_{0}\right)}{\log t} \lim _{t \rightarrow+\infty} \frac{\log t}{\psi(t)}=a_{\psi},
$$

a formula which is also true if $a_{\psi}=+\infty$.

In the locally finite tree case, Corollary 1.2 of [HP5] applies directly, since it proves that $\lim \sup _{t \rightarrow+\infty} \frac{d\left(v_{-t}, \Gamma v_{0}\right)}{\log t}=\frac{1}{\delta}$ for almost every $v \in T^{1} X$ for the BowenMargulis measure. 
Assume hence that $X$ is a Riemannian manifold as in the statement. The only assumption of Corollary 6.1 of [HP4] that is not an assumption of Corollary 12 is that there exists $c^{\prime}>0$ such that $\frac{1}{c^{\prime}} e^{\delta n} \leq \operatorname{Card}\left\{\gamma \in \Gamma: d\left(x_{0}, \gamma x_{0}\right) \leq n\right\} \leq c^{\prime} e^{\delta n}$ for all $n \in \mathbb{N}$.

By [DOP], the assumptions that $\Gamma$ is geometrically finite and that $\delta_{p}<\delta$ for all $p \in \operatorname{Par}_{\Gamma}$ imply the finiteness of the Bowen-Margulis measure on $\Gamma \backslash T^{1} X$. By [Dal] since $\operatorname{Par}_{\Gamma} \neq \emptyset$, the set of the translation lengths of the hyperbolic elements of $\Gamma$ is not contained in a discrete subgroup of $\mathbb{R}$. By [Rob], the extra assumption above is satisfied (and there is even an asymptotic equivalent $\operatorname{Card}\left\{\gamma \in \Gamma: d\left(x_{0}, \gamma x_{0}\right) \leq\right.$ $n\} \sim c^{\prime} e^{\delta n}$ as $\left.n \rightarrow+\infty\right)$. Since the conclusion of Corollary 6.1 of [HP4] is that $\lim \sup _{t \rightarrow+\infty} \frac{d\left(v_{-t}, \Gamma v_{0}\right)}{\log t}=\frac{1}{2\left(\delta-\delta_{0}\right)}$ for almost every $v \in T^{1} X$ for the BowenMargulis measure, Corollary 12 follows from Equation (7).

Remark. Since Corollary 6.1 of [HP4] and Corollary 1.2 of [HP5] are valid cusp by cusp, a statement analogous to Corollary 12 for a prescribed set of cusps is also valid.

As a second application of our main theorem, here is another consequence, for the behavior of strong unstable leaves, of the excursion properties of the geodesic flow in cusps neighborhoods.

Corollary 13. If $\Gamma$ is convex-cocompact, then for every $v \in T^{1} X$ such that $v_{-} \in \Lambda \Gamma$, we have $\lim \sup _{s \rightarrow+\infty} \frac{\Theta_{v}(s)}{\log s}=1$.

If $\Gamma$ is not convex-cocompact and if $X$ is a Riemannian manifold of dimension at least 3, then for every $\alpha \in[1,2]$, there exists $v \in T^{1} X$ such that

$$
\limsup _{s \rightarrow+\infty} \frac{\Theta_{v}(s)}{\log s}=\alpha
$$

Proof. The first claim is immediate from Theorem 7 with $\psi: t \mapsto t$. The second one follows from the techniques of $§ 5.4$ of [PP1].

Given $\alpha \in] 1,2]$, it would be interesting to study the Hausdorff dimension of the set of elements $v \in T^{1} X$ such that $\lim \sup _{s \rightarrow+\infty} \frac{\Theta_{v}(s)}{\log s}=\alpha$.

\section{An application to non-Archimedian Diophantine approximation}

For all $n \geq 2$, let $\mathbb{H}_{\mathbb{R}}^{n}$ be the upper half-space model of the real hyperbolic space of dimension $n$. Applications to Archimedian Diophantine approximation may be obtained, as in the case of $X=\mathbb{H}_{\mathbb{R}}^{2}$ and $\Gamma$ a congruence subgroup of $\mathrm{PSL}_{2}(\mathbb{Z}$ ) (see for instance $[\mathrm{AM}]$ ), by taking for instance $X=\mathbb{H}_{\mathbb{R}}^{3}$ and $\Gamma=\operatorname{PSL}(\mathscr{O})$ where $\mathscr{O}$ is an order in the ring of integers of an imaginary quadratic number field, or $X=\mathbb{H}_{\mathbb{R}}^{5}$ 
and $\Gamma=\operatorname{PSL}(\mathscr{O})$ where $\mathscr{O}$ is an order in a definite quaternion algebra over $\mathbb{Q}$ (see for instance [PP2]). But in this paper, we concentrate on the applications to non-Archimedian Diophantine approximation.

We start this section by restating a version of Theorem 11 in the particular case of trees, which will be more directly applicable for our arithmetic applications.

Let $T$ be a locally finite tree (endowed with the maximal distance making each edge isometric to $[0,1]$, which is $\operatorname{CAT}(-1))$. Let $\mathrm{V}(T)$ be its set of vertices and $\operatorname{Aut}(T)$ its locally compact automorphism groups (which is contained in its isometry group). Let $\Gamma$ be a geometrically finite subgroup of $\operatorname{Aut}(T)$. Up to taking the first barycentric subdivision of $T$ and rescaling, we assume that $\Gamma$ acts without inversion (that is, no element of $\Gamma$ maps an edge of $T$ to its opposite edge), so that $\Gamma \backslash T$ has a unique structure of graph such that the canonical projection $T \rightarrow \Gamma \backslash T$ is a morphism of graphs. By the structure theorem of [Pau2] (improving on the algebraic cases of Serre [Ser] and Lubotzky [Lub]), with $E$ the finite set $\Gamma \backslash \operatorname{Par}_{\Gamma}$, there exist a finite subgraph $\mathscr{G}$ of $\Gamma \backslash \mathscr{C} \Lambda \Gamma$ and for every $e \in E$, a geodesic ray $\rho_{e}:[0,+\infty[\rightarrow$ $\Gamma \backslash \mathscr{C} \Lambda \Gamma$ with origin a vertex, which lifts to a geodesic ray in $T$ converging to any representative of $e$ in $\operatorname{Par}_{\Gamma}$, such that $\Gamma \backslash \mathscr{C} \Lambda \Gamma$ is the disjoint union of $\mathscr{G}$ and the open rays $\rho_{e}(] 0,+\infty[)$ for $e \in E$.

For every $e \in E$, define a map $\Delta_{e}: \mathrm{V}(T) \rightarrow\left[0,+\infty\left[\right.\right.$ by $\Delta_{e}(x)=n$ if $\Gamma x=$ $\rho_{e}(n)$ (such an $n$ is unique if it exists), and $\Delta_{e}(x)=0$ otherwise. Note that if $\tilde{e}$ is an element of $\operatorname{Par}_{\Gamma}$ whose image in $\Gamma \backslash \operatorname{Par}_{\Gamma}$ is $e$, since $\rho_{e}$ lifts to a geodesic ray converging to $\tilde{e}$, there exists a constant $c \in \mathbb{R}$ such that $\tilde{\beta}_{\{\tilde{e}\}}(t)=\Delta_{e}(t)+c$ for $t$ big enough, with the notation before Theorem 11. Also note that two geodesic lines in $T$, starting from the same point at infinity, coincide up to translation on a neighborhood of $-\infty$. For every non-isolated point $\xi_{*} \in \partial_{\infty} T$, we endow $\partial_{\infty} T-\left\{\xi_{*}\right\}$ with the filter of the complementary subsets of its relatively compact subsets. Therefore, the following result follows immediately from the definition of Hamenstädt's distance and Theorem 11.

Corollary 14. Let $\xi_{*} \in \Lambda_{c} \Gamma$ and $\eta_{*} \in \partial_{\infty} T-\left\{\xi_{*}\right\}$. For every $\eta \in \partial_{\infty} T-\left\{\xi_{*}\right\}$, let $t \mapsto \eta(t)$ be the geodesic line from $\xi_{*}$ to $\eta$ such that $\eta_{*}(0) \in \mathrm{V}(T)$ and $\eta(t)=\eta_{*}(t)$ for $t$ small enough. Let $\delta_{*}\left(\eta, \eta_{*}\right)=\inf \left\{t \in \mathbb{N}: \eta(-s)=\eta_{*}(-s)\right.$ for all $\left.s \geq t\right\}$. Then for all $e \in E$ and $\psi:] 0,+\infty[\rightarrow] 0,+\infty[$ slowly increasing, we have

$$
\limsup _{\eta \in \partial_{\infty} T-\left\{\xi_{*}\right\}} \frac{\Delta_{e}(\eta(0))}{\psi\left(\delta_{*}\left(\eta, \eta_{*}\right)\right)}=a_{\psi}+\limsup _{t \rightarrow+\infty} \frac{\Delta_{e}\left(\eta_{*}(-t)\right)}{\psi(t)} .
$$

We now give our applications to non-Archimedian Diophantine approximation. We follow the notation of [Pau1], in particular as recalled in the introduction for $k=\mathbb{F}_{q}, A=k[X], K=k(X), \widehat{K}=k\left(\left(X^{-1}\right)\right), \mathscr{O}=k\left[\left[X^{-1}\right]\right],|\cdot|=|\cdot|_{\infty}$ and, for every $f \in \widehat{K}-K$, its continued fraction expansion $\left(a_{n}=a_{n}(f)\right)_{n \in \mathbb{N}}$ and its approximation exponent $v=v(f)$. 
For every $f=\sum_{i \in \mathbb{Z}} f_{i} X^{-i} \in \widehat{K}$, the integral part $[f]$ of $f$ is $\sum_{i \leq 0} f_{i} X^{-i} \in A$ and its fractional part $\{f\}$ is $\sum_{i>0} f_{i} X^{-i} \in X^{-1} \mathscr{O}$. Artin's map $\Psi: X^{-1} \mathscr{O}-\{0\} \rightarrow$ $X^{-1} \mathscr{O}$ is defined by $f \mapsto\{1 / f\}$. Given $f \in \widehat{K}-K$, we have $a_{0}=[f]$ and if $n \geq 1$, then $a_{n}=\left[\frac{1}{\psi^{n-1}\left(f-a_{0}\right)}\right]$. Consider the sequences $\left(P_{n}\right)_{n \in \mathbb{N} \cup\{-1\}}$ and $\left(Q_{n}\right)_{n \in \mathbb{N} \cup\{-1\}}$ in $A$ inductively defined by

$$
P_{-1}=1, \quad Q_{-1}=0, \quad P_{0}=a_{0}, \quad Q_{0}=1
$$

and for every $n \in \mathbb{N}$,

$$
P_{n+1}=a_{n+1} P_{n}+P_{n-1} \quad \text { and } \quad Q_{n+1}=a_{n+1} Q_{n}+Q_{n-1} .
$$

Then $P_{n}$ and $Q_{n}$ are relatively prime, and

$$
\frac{P_{n}}{Q_{n}}=a_{0}+\frac{1}{a_{1}+\frac{1}{a_{2}+\frac{1}{\ddots}}}
$$

is called the $n$-th convergent of $f$. The sequence $\left(\frac{P_{n}}{Q_{n}}\right)_{n \in \mathbb{N}}$ converges to $f$ (for the above, see for instance [Las], [Sch], as well as [Paul] for a geometric explanation).

The action of $\mathrm{GL}_{2}(\widehat{K})$ on the set of $\mathscr{O}$-lattices induces an action of $\widehat{K}^{*}$ by homotheties on this set, and we will denote by $[\Lambda]$ the homothety class of an $\mathscr{O}$-lattice $\Lambda$.

Remarks. (1) Note that $\mathscr{O}$-lattices $\Lambda$ in $\widehat{K}^{2}$ behave, from the topological viewpoint, very differently than $\mathbb{Z}$-lattices in $\mathbb{R}^{2}$ : they are compact open additive subgroups of $\widehat{K}^{2}$, and hence $\widehat{K}^{2} / \Lambda$ is infinite and discrete (thus non-compact). Furthermore, for any norm $\|\cdot\|$ on $\widehat{K}^{2}$, we have $\inf _{x \in \Lambda-\{0\}}\|x\|=0$.

(2) The set $\mathrm{V}\left(\mathbb{T}_{\tilde{K}}\right)$ (see below for an explanation of this notation) of homothety classes of $\mathscr{O}$-lattices in $\widehat{K}^{2}$ can be endowed with the quotient of Chabauty's topology on closed subgroups of the (additive) locally compact group $\widehat{K}^{2}$, or, equivalently, with the topology of an homogeneous space under the transitive (linear) action of $\mathrm{PGL}_{2}(\hat{K})$. Note that this topology is discrete since $\mathrm{PGL}_{2}(\mathscr{O})$ is open in $\mathrm{PGL}_{2}(\hat{K})$, again a major difference from the case of $\mathbb{Z}$-lattices in $\mathbb{R}^{2}$. The map $\Delta$ defined in the introduction induces (by passing to the quotients) a proper map from $\operatorname{PSL}_{2}(A) \backslash \mathrm{V}\left(\mathbb{T}_{\tilde{K}}\right)$ to $\mathbb{N}$. This map is an ultrametric analog of the inverse of the systole map on $\mathbb{Z}$-lattices with covolume 1 in $\mathbb{R}^{2}$, whose properness is called Mahler's criterion.

Let us fix a nonzero element $Q_{*}$ of $A$. Consider Hecke's nonprincipal congruence subgroup

$$
\Gamma_{Q_{*}}^{0}=\left\{\left(\begin{array}{ll}
a & b \\
c & d
\end{array}\right) \in \operatorname{SL}_{2}(A): c \equiv 0 \bmod Q_{*}\right\},
$$


which has finite index in $\operatorname{SL}_{2}(A)$. For every $\mathscr{O}$-lattice $\Lambda$, define $\Delta_{Q_{*}}(\Lambda)=n$ if there exists $\gamma \in \Gamma_{Q_{*}}^{0}$ such that $[\gamma \Lambda]=\left[\mathscr{O} \times X^{-n} \mathscr{O}\right]$ and $\Delta_{Q_{*}}(\Lambda)=0$ otherwise.

For every $f \in \widehat{K}-K$, recall that $\left(u_{g}=u_{g}(f)\right)_{g \in \hat{K}}$ is the maximal one-parameter unipotent subgroup of $\mathrm{SL}_{2}(\hat{K})$ whose projective action on $\mathbb{P}_{1}(\hat{K})=\hat{K} \cup\{\infty\}$ fixes $f$. For every $f \in \hat{K}-K$, the approximation exponent $v_{Q_{*}}=v_{Q_{*}}(f)$ of $f$ relative to $Q_{*}$ is the least upper bound of the positive numbers $v^{\prime}$ such that there exist infinitely many elements $\frac{P}{Q}$ in $K$ with $P$ and $Q$ relatively prime and $Q \equiv 0 \bmod Q_{*}$ such that

$$
\left|f-\frac{P}{Q}\right| \leq|Q|^{-v^{\prime}}
$$

Theorem 15. For every $f \in \widehat{K}-K$, we have

$$
\begin{aligned}
\limsup _{|g| \rightarrow+\infty} \frac{\Delta_{Q_{*}}\left(u_{g} \mathscr{O}^{2}\right)}{\log _{q}|g|} & =2-\frac{2}{v_{Q_{*}}} \\
& =1+\limsup _{n \rightarrow+\infty: Q_{n} \equiv 0 \bmod Q_{*}} \frac{\log \left|a_{n+1}\right|}{\log \left|a_{n+1} \prod_{i=1}^{n} a_{i}^{2}\right|} .
\end{aligned}
$$

Theorem 3 in the introduction follows by taking $Q_{*}=1$.

Proof. We will apply Corollary 14 with $T$ the Bruhat-Tits tree of $\left(\mathrm{PGL}_{2}, \hat{K}\right)$, whose definition and useful properties we start by recalling (following [Ser]).

The Bruhat-Tits tree $\mathbb{T}_{\hat{K}}$ of $\left(\mathrm{PGL}_{2}, \widehat{K}\right)$ is the graph whose vertices are the homothety classes of $\mathscr{O}$-lattices in $\widehat{K}^{2}$, two vertices $x$ and $x^{\prime}$ being joined by an edge if and only if there exist representatives $\Lambda, \Lambda^{\prime}$ of $x, x^{\prime}$ respectively such that $\Lambda^{\prime} \subset \Lambda$ and $\Lambda / \Lambda^{\prime}$ is isomorphic to $\mathscr{O} / X^{-1} \mathscr{O}$.

We identify as usual the projective line $\mathbb{P}_{1}(\hat{K})$ with $\widehat{K} \cup\{\infty\}$ by the map

$$
\widehat{K}^{*}(x, y) \mapsto \frac{x}{y} .
$$

We denote by $(g, x) \mapsto g \cdot x$ the projective action of $g \in \mathrm{GL}_{2}(\widehat{K})$ on $x \in \mathbb{P}_{1}(\widehat{K})=$ $\widehat{K} \cup\{\infty\}$.

The action of $\mathrm{GL}_{2}(\hat{K})$ on the set of $\mathscr{O}$-lattices induces an isometric action of $\mathrm{GL}_{2}(\hat{K})$ on $\mathbb{T}_{\hat{K}}$. Note that $\mathrm{SL}_{2}(\hat{K})$ acts with two orbits on the set of vertices of $\mathbb{T}_{\hat{K}}$. There exists one and only one homeomorphism between $\partial_{\infty} \mathbb{T}_{\hat{K}}$ and $\mathbb{P}_{1}(\hat{K})$ such that the (continuous) extension to $\partial_{\infty} \mathbb{T}_{\widehat{K}}$ of the isometric action of $\mathrm{GL}_{2}(\widehat{K})$ on $\mathbb{T}_{\widehat{K}}$ corresponds to the projective action of $\mathrm{GL}_{2}(\widehat{K})$ on $\mathbb{P}_{1}(\widehat{K})$. From now on, we identify $\partial_{\infty} \mathbb{T}_{\widehat{K}}$ and $\mathbb{P}_{1}(\hat{K})$ by this homeomorphism.

We denote by $\mathrm{HB}_{\infty}$ the horoball in $\mathbb{T}_{\widehat{K}}$ with center $\infty$ whose boundary contains the vertex $\left[\mathscr{O}^{2}\right]$. Note that $\operatorname{PSL}_{2}(A)$ is a geometrically finite group of isometries of $\mathbb{T}_{\widehat{K}}$, with only one orbit of parabolic points, and that $\left(\gamma \mathrm{HB}_{\infty}\right)_{\gamma \in \mathrm{SL}_{2}(A) / \mathrm{SL}_{2}(A)_{\infty}}$ is the 
associated $\operatorname{PSL}_{2}(A)$-equivariant family of maximal horoballs with pairwise disjoint interiors (see $\S 6.2$ of [Pau1]). The geodesic ray from $\left[\mathscr{O}^{2}\right]$ to $\infty \in \partial_{\infty} \mathbb{T}_{\hat{K}}$, whose sequence of consecutive vertices is $\left(\left[\mathscr{O} \times X^{-n} \mathscr{O}\right]\right)_{n \in \mathbb{N}}$, injects onto the quotient $\operatorname{PSL}_{2}(A) \backslash \mathbb{T}_{\widehat{K}}$.

Let us fix $f \in \hat{K}-K$. We are now going to apply Corollary 14 with $\psi=$ id (so that $a_{\psi}=1$ ), $T=\mathbb{T}_{\widehat{K}}, \Gamma$ the image of $\Gamma_{Q_{*}}^{0}$ in $\operatorname{Aut}(T)$ (which is also a geometrically finite subgroup with $\operatorname{Par}_{\Gamma}=\operatorname{Par}_{\mathrm{PSL}_{2}(A)}=\mathbb{P}_{1}(K)=K \cup\{\infty\}$ ), $\xi_{*}=f$ (which is a conical limit point, since $f$ is irrational and the limit set of $\operatorname{PSL}_{2}(A)$, hence of $\Gamma$, is the whole boundary at infinity), $\eta_{*}=0$, and $e=\Gamma \infty$.
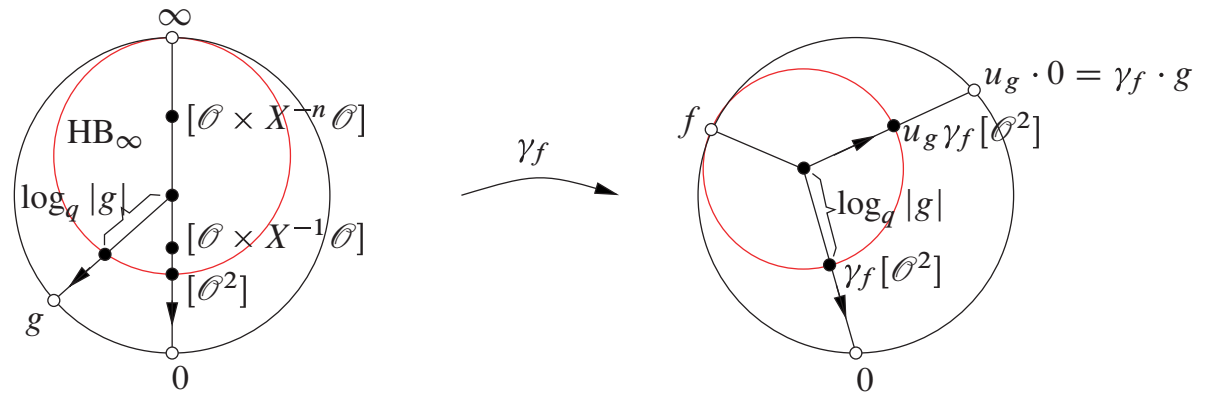

The sequence $\left(\left[\mathscr{O} \times X^{-n} \mathscr{O}\right]\right)_{n \in \mathbb{N}}$ of consecutive vertices of the geodesic ray in $\mathbb{T}_{\widehat{K}}$ from $\left[\mathscr{O}^{2}\right]$ to $\infty$ isometrically injects in $\operatorname{PSL}_{2}(A) \backslash \mathbb{T}_{\widehat{K}}$, hence in $\Gamma_{Q_{*}}^{0} \backslash \mathbb{T}_{\widehat{K}}$. Therefore, there exists a constant $c \in \mathbb{R}$ such that for every $\mathscr{O}$-lattice $\Lambda$, we have

$$
\left|\Delta_{e}([\Lambda])-\Delta_{Q_{*}}(\Lambda)\right| \leq c .
$$

The element $\gamma_{f}=\left(\begin{array}{cc}1 & 0 \\ 1 / f & 1\end{array}\right) \in \mathrm{SL}_{2}(\widehat{K})$ maps projectively $\infty$ to $f$ (hence sends the horospheres centered at $\infty$ to the horospheres centered at $f$ ) and fixes 0 . The maximal one-parameter unipotent subgroup of $\mathrm{SL}_{2}(\widehat{K})$ fixing $\infty$ is $g \mapsto\left(\begin{array}{ll}1 & g \\ 0 & 1\end{array}\right)$. Hence the maximal one-parameter unipotent subgroup $\left(u_{g}\right)_{g \in \hat{K}}$ of $\operatorname{SL}_{2}(\hat{K})$ fixing $f$ is $g \mapsto u_{g}=\gamma_{f}\left(\begin{array}{ll}1 & g \\ 0 & 1\end{array}\right) \gamma_{f}^{-1}$. We fix the parametrization of the geodesic lines starting from $f$ so that they cross at time $t=0$ through $\gamma_{f} \partial \mathrm{HB}_{\infty}$. Note that there exists a constant $c^{\prime} \in \mathbb{R}$ such that for all $g \in \hat{K}$, we have

$$
\left|\Delta_{Q_{*}}\left(u_{g} \gamma_{f} \mathscr{O}^{2}\right)-\Delta_{Q_{*}}\left(u_{g} \mathscr{O}^{2}\right)\right| \leq c^{\prime} .
$$

By Corollary 5.2 of [Pau1], for all $g \in \hat{K}$, Hamenstädt's distance between the geodesic lines starting from $\infty$ and ending at 0 and at $g$, passing through $\partial \mathrm{HB}_{\infty}$ at time 0 , is $|g|^{\frac{1}{\log q}}$. Since $\gamma_{f}$ is an isometry fixing 0 and $\gamma_{f} \cdot g=u_{g} \cdot 0$, with $\delta_{*}(\cdot, \cdot)$ defined in Corollary 14, we hence have

$$
\delta_{*}\left(u_{g} \cdot 0,0\right)=\log _{q}|g| .
$$


The map from $\hat{K}$ to $\partial_{\infty} \mathbb{T}_{\hat{K}}-\{f\}$ sending $g$ to $\eta=u_{g} \cdot 0$ is a homeomorphism. With $t \mapsto \eta(t)$ as defined in Corollary 14, we may assume that $\eta_{*}(0)=\gamma_{f}\left[\mathscr{O}^{2}\right]$ (this changes $\Delta_{e}(\eta(0))$ only by a constant), and we have $\eta(0)=u_{g} \gamma_{f}\left[\mathscr{O}^{2}\right]$ (see the above picture on the right). Hence

$$
\limsup _{\eta \in \partial_{\infty} T-\left\{\xi_{*}\right\}} \frac{\Delta_{e}(\eta(0))}{\delta_{*}\left(\eta, \eta_{*}\right)}=\limsup _{|g| \rightarrow+\infty} \frac{\Delta_{Q_{*}}\left(u_{g} \mathscr{O}^{2}\right)}{\log _{q}|g|} .
$$

Let us denote by $v_{\infty}: \hat{K} \rightarrow \mathbb{Z} \cup\{+\infty\}$ the valuation associated to the absolute value $|\cdot|$, so that for all $f \in \widehat{K}$, we have

$$
|f|=q^{-v_{\infty}(f)} .
$$

By $\S 6.3$ of [Pau1] the geodesic line starting from $\infty$ and ending at $f$ (passing at time $t=0$ through $\partial \mathrm{HB}_{\infty}$ ) enters successively the interiors of the horoballs of the $\operatorname{PSL}_{2}(A)$-equivariant family of maximal horoballs with pairwise disjoint interiors which are centered at the convergents of $f$. Furthermore, if $x_{n}$ is its entering vertex in $\mathrm{HB}_{\frac{P_{n}}{Q_{n}}}$ and $y_{n}$ its point the closest to $\frac{P_{n}}{Q_{n}}$, then

$$
d\left(x_{n}, y_{n}\right)=\frac{1}{2} d\left(x_{n}, x_{n+1}\right)=-v_{\infty}\left(a_{n+1}\right) .
$$

In particular,

$$
d\left(x_{0}, y_{n}\right)=\sum_{i=1}^{n}-2 v_{\infty}\left(a_{i}\right)-v_{\infty}\left(a_{n+1}\right)=\log _{q}\left|a_{n+1} \prod_{i=1}^{n} a_{i}^{2}\right| .
$$

By the definition of $\Delta_{Q_{*}}$, we are only interested in the excursions of the geodesic line ] $\infty, f$ in the horospheres $\mathrm{HB}_{\frac{P}{Q}}$ with $P, Q \in A$ relatively prime and $Q=$ $0 \bmod Q_{*}$. Since the geodesic ray from $\gamma_{f}\left[\mathscr{O}^{2}\right]$ to $f$ coincides, for times big enough and up to translation, with the geodesic line $] \infty, f[$, we have

$$
\limsup _{t \rightarrow+\infty} \frac{\Delta_{e}\left(\eta_{*}(-t)\right)}{t}=\limsup _{n \rightarrow+\infty: Q_{n}=0 \bmod Q_{*}} \frac{\log _{q}\left|a_{n+1}\right|}{\log _{q}\left|a_{n+1} \prod_{i=1}^{n} a_{i}^{2}\right|} .
$$

By $\S 6.3$ of [Pau1] with the above notation, we also have

$$
d\left(x_{0}, y_{n}\right)=v_{\infty}\left(f-\frac{P_{n}}{Q_{n}}\right)=-\log _{q}\left|f-\frac{P_{n}}{Q_{n}}\right|,
$$

and

$$
d\left(x_{0}, x_{n}\right)=-2 v_{\infty}\left(Q_{n}\right)=2 \log _{q}\left|Q_{n}\right|
$$


Since $\left|f-\frac{P}{Q}\right|$ is at least 1 if $\frac{P}{Q}$ is not a convergent of $f$, we hence have, by the definition of the approximation exponent $v_{Q_{*}}$,

$$
\begin{aligned}
\frac{2}{v_{Q_{*}}} & =\liminf _{|Q| \rightarrow+\infty: Q \equiv 0 \bmod Q_{*}} \frac{2 \log |Q|}{-\log \left|f-\frac{P}{Q}\right|} \\
& =\liminf _{n \rightarrow+\infty: Q_{n} \equiv 0 \bmod Q_{*}} \frac{2 \log _{q}\left|Q_{n}\right|}{-\log _{q}\left|f-\frac{P_{n}}{Q_{n}}\right|} \\
& =\liminf _{n \rightarrow+\infty: Q_{n} \equiv 0 \bmod Q_{*}} \frac{d\left(x_{0}, x_{n}\right)}{d\left(x_{0}, y_{n}\right)} \\
& =\liminf _{n \rightarrow+\infty: Q_{n} \equiv 0 \bmod Q_{*}} \frac{\sum_{i=1}^{n}-2 v_{\infty}\left(a_{i}\right)}{\sum_{i=1}^{n}-2 v_{\infty}\left(a_{i}\right)-v_{\infty}\left(a_{n+1}\right)} \\
& =1-\limsup _{n \rightarrow+\infty: Q_{n} \equiv 0 \bmod Q_{*}} \frac{\log _{q}\left|a_{n+1}\right|}{\log _{q}\left|a_{n+1} \prod_{i=1}^{n} a_{i}^{2}\right|} .
\end{aligned}
$$

Now Theorem 15 follows from Equation (8), Corollary 14, Equation (9), and Equation (10).

Remark. Note that the definition of $\Delta_{Q_{*}}$ is related to the choice of one of the ends of $\Gamma_{Q_{*}}^{0} \backslash \mathbb{T}_{\widehat{K}}$ (the one corresponding to the geodesic ray in $\mathbb{T}_{\widehat{K}}$ with vertices $\left[\mathscr{O} \times X^{-n} \mathscr{O}\right]$ for $n \in \mathbb{N}$ ). Other choices of ends give analogous Diophantine approximation results.

\section{References}

[Ath] J. Athreya, Cusp excursions on parameter spaces. J. London Math. Soc. (2) 87 (2013), no. 3, 741-765. Zbl 1277.37006 MR 3073674

[AGP1] J. Athreya, A. Ghosh, and A. Prasad, Ultrametric logarithm law, I. Discrete Contin. Dyn. Syst. Ser. S 2 (2009), no. 2, 337-348. Zbl 1175.37009 MR 2505643

[AGP2] J. Athreya, A. Ghosh, and A. Prasad, Ultrametric logarithm laws, II. Monatsh. Math. 167 (2012), no. 3-4, 333-356 Zbl 06093472 MR 2961287

[AM] J. Athreya and G. Margulis, Logarithm laws for unipotent flows, I. J. Modern Dyn. 3 (2009), no. 3, 359-378. Zbl 1184.37007 MR 2538473

[Bow] B. Bowditch, Geometrical finiteness with variable negative curvature. Duke Math. J. 77 (1995), no. 1, 229-274. Zbl 0877.57018 MR 1317633

[BH] M. R. Bridson and A. Haefliger, Metric spaces of non-positive curvature. Grundlehren Math. Wiss. 319, Springer Verlag, Berlin 1999. Zbl 0988.53001 MR 1744486

[BK] P. Buser and H. Karcher, Gromov's almost flat manifolds. Astérisque 81, Soc. Math. France, Paris 1981. Zbl 0459.53031 MR 0619537

[Dal] F. Dal'Bo, Remarques sur le spectre des longueurs d'une surface et comptage. Bol. Soc. Brasil. Mat. (N.S.) 30 (1999), no. 2, 199-221. Zbl 1058.53063 MR 1703039 
[DOP] F. Dal'Bo, J.-P. Otal, and M. Peigné, Séries de Poincaré des groupes géométriquement finis. Israel J. Math. 118 (2000), 109-124. Zbl 0968.53023 MR 1776078

[EFJ] N. Enriquez, J. Franchi, and Y. Le Jan, Stable windings on hyperbolic surfaces. Probab. Theory Related Fields 119 (2001), no. 2, 213-255. Zbl 1172.37311 MR 1818247

[Esk] A. Eskin, Unipotent flows and applications. In Homogeneous flows, moduli spaces, and arithmetic, Clay Math. Inst. Summer School (Pisa 2007), Clay Math. Inst. Proc. 10, Amer. Math. Soc., Providence, RI, 2010, 71-129. Zbl 1243.37004 MR 2648693

[Ham] U. Hamenstädt, A new description of the Bowen-Margulis measure. Ergodic Theory Dynam. Systems 9 (1989), no. 3, 455-464. Zbl 0722.58029 MR 1016663

[HP1] S. Hersonsky and F. Paulin, On the rigidity of discrete isometry groups of negatively curved spaces. Comment. Math. Helv. 72 (1997), no. 3, 349-388. Zbl 0908.57009 MR 1476054

[HP2] S. Hersonsky and F. Paulin, Diophantine approximation for negatively curved manifolds. Math. Z. 241 (2002), no. 1, 181-226. Zbl 1037.53020 MR 1930990

[HP3] S. Hersonsky and F. Paulin, Diophantine approximation in negatively curved manifolds and in the Heisenberg group. In Rigidity in dynamics and geometry (Cambridge, 2000), M. Burger, A. Iozzi (eds.), Springer Verlag, Berlin 2002, 203-226. Zbl 1064.11057 MR 1919402

[HP4] S. Hersonsky and F. Paulin, Counting orbit points in coverings of negatively curved manifolds and Hausdorff dimension of cusp excursions. Ergodic Theory Dynam. Systems 24 (2004), no. 3, 803-824. Zbl 1059.37022 MR 2060999

[HP5] S. Hersonsky and F. Paulin, A logarithm law for automorphism groups of trees. Arch. Math. 88 (2007), no. 2, 97-108. Zbl 1160.20020 MR 2299033

[HP6] S. Hersonsky and F. Paulin, On the almost sure spiraling of geodesics in negatively curved manifolds. J. Differential Geom. 85 (2010), no. 2, 271-314. Zbl 1229.53050 MR 2732978

[KeM] D. Kelmer and A. Mohammadi, Logarithm laws for one-parameter unipotent flows. Geom. Funct. Anal. 22 (2012), no. 3, 756-784. Zbl 1260.37003 MR 2972608

[KIM] D. Kleinbock and G. Margulis, Logarithm laws for flows on homogeneous spaces. Invent. Math. 138 (1999), no. 3, 451-494. Zbl 0934.22016 MR 1719827

[Las] A. Lasjaunias, A survey of Diophantine approximation in fields of power series. Monatsh. Math. 130 (2000), no. 3, 211-229. Zbl 0990.11043 MR 1777096

[Lub] A. Lubotzky, Lattices in rank one Lie groups over local fields. Geom. Funct. Anal. 1 (1991), no. 4, 406-431. Zbl 0786.22017 MR 1132296

[PP1] J. Parkkonen and F. Paulin, Prescribing the behaviour of geodesics in negative curvature. Geom. Topol. 14 (2010), no. 1, 277-392. Zbl 1191.53026 MR 2578306

[PP2] J. Parkkonen and F. Paulin, On the arithmetic and geometry of binary Hamiltonian forms. With an appendix by V. Emery. Algebra Number Theory 7 (2013), no. 1, 75115. Zbl 1273.11065 MR 3037891

[Pau1] F. Paulin, Groupe modulaire, fractions continues et approximation diophantienne en caractéristique p. Geom. Dedicata 95 (2002), 65-85. Zbl 1033.20026 MR 950885 
[Pau2] F. Paulin, Groupes géométriquement finis d'automorphismes d'arbres et approximation diophantienne dans les arbres. Manuscripta Math. 113 (2004), no. 1, 1-23. Zbl 1070.20032 MR 2135558

[PPS] F. Paulin, M. Pollicott, and B. Schapira, Equilibrium states in negative curvature. Book preprint, arXiv:1211.6242, hal-00758114; Astérisque, Soc. Math. France, to appear.

[Rob] T. Roblin, Ergodicité et équidistribution en courbure négative. Mém. Soc. Math. Fr. (N.S.) No. 95 (2003). Zbl 1056.37034 MR 2057305

[Sch] W. Schmidt, On continued fractions and diophantine approximation in power series fields. Acta Arith. 95 (2000), no. 2, 139-166. Zbl 0987.11041 MR 1785412

[Ser] J.-P. Serre, Arbres, amalgames, $S L_{2}$. 3ème éd. corr., Astérisque 46, Soc. Math. France, Paris 1983. Zbl 0369.20013 MR 476875

[SV] B. Stratmann and S. L. Velani, The Patterson measure for geometrically finite groups with parabolic elements, new and old. Proc. London Math. Soc. 71 (1995), no. 1, 197-220. Zbl 0821.58026 MR 1327939

[Sul] D. Sullivan, Disjoint spheres, approximation by imaginary quadratic numbers, and the logarithm law for geodesics. Acta Math. 149 (1982), no. 3-4, 215-237. Zbl 0517.58028 MR 0688349

Received May 18, 2012; revised February 11, 2013

J. S. Athreya, Department of Mathematics, University of Illinois Urbana-Champaign, 1409 W. Green Street, Urbana, IL 61801, U.S.A.

E-mail: jathreya@illinois.edu

F. Paulin, Département de mathématique, UMR 8628 CNRS, Bâtiment 425, Université

Paris-Sud, 91405 Orsay Cedex, France

E-mail: frederic.paulin@math.u-psud.fr 